\title{
Escala diagramática para avaliação da mancha marrom de alternaria de citros
}

\author{
Marisa Silveira Almeida Renaud ${ }^{1}$, Lilian Amorim $^{1}$, Silvia Afonseca Lourenço ${ }^{1}$, Marcel Bellato Spósito
}

${ }^{1}$ Departamento de Entomologia, Fitopatologia e Zoologia Agrícola, ESALQ/USP, CP 09, 13418-900, Piracicaba, SP. ${ }^{2}$ Fundecitrus, Av. Adhemar P. de Barros, 201, 14807-040, Araraquara, SP. E-mail: msarenaud@yahoo.com.br

Autor para correspondência: Marisa Silveira Almeida Renaud. msarenaud@yahoo.com.br

Data de chegada: 13/12/2005. Aceito para publicação em: 28/05/2008

\section{RESUMO}

Renaud, M.S.A., Amorim, L., Lourenço, S.A., Spósito, M.B. Escala diagramática para avaliação da mancha marrom de alternaria de citros. Summa Phytopathologica, v.34, n.3, p.270-271, 2008

Com o objetivo de padronizar as avaliações da severidade da Mancha Marrom de Alternaria, foi desenvolvida uma escala diagramática considerando a distribuição, a forma, a freqüência das lesões e os níveis mínimo e máximo de severidade encontrados em campo. Dois tipos de lesões comuns em campo foram representados na escala, pequenas de formato circular ou irregular $(0,1 ; 1 ; 2,5 ; 5 ; 11$ e $25 \%$ da área do fruto lesionada) e grandes, nesse caso sempre circulares $(1 ; 2,5 ; 5 ; 10$ e $17 \%$ da área do fruto lesionada). A validação da escala foi feita em duas etapas: na primeira, quatro avaliadores estimaram a severidade de 79 fotos de frutos sintomáticos, sem o uso da escala, e na segunda, os avaliadores estimaram a severidade dos mesmos frutos com o uso da escala. Quando a escala não foi utilizada, o $r^{2}$ estimado para todo o conjunto de dados foi 0,69 , o intercepto (a) 1,42 e o coeficiente angular (b) 0,77. Com o uso da escala, esses parâmetros foram de $0,84,1,59$ e 0,84 , respectivamente. A escala mostrou-se adequada para avaliações da severidade da Mancha Marrom de Alternaria em frutos cítricos.

Palavras chave adicionais: Alternaria alternata, patometria, avaliação de doenças

\section{ABSTRACT}

Renaud, M.S.A., Amorim, L., Lourenço, S.A., Spósito, M.B. Diagrammatic scale for assessment of Alternaria Brown spot of citrus. Summa Phytopathologica, v.34, n.3, p.270-271, 2008

A diagrammatic scale of Alternaria Brown Spot in citrus was developed in order to standardize disease severity assessments. The scale was built taking into consideration the distribution, shape and frequency of lesions as well as minimum and maximum severity levels found in the field. Two types of symptoms widely observed at field were represented in the diagrammatic scale: small circular or irregular lesions (l0.1 12.5511 and $25 \%$ ) and large circular lesions (1 2.5510 and 17\%). The validation of the scale was accomplished in two stages. Firstly, four experts estimated the severity of disease in 79 photographs of citrus fruits with symptoms without using the diagrammatic scale. Secondly, the experts estimated the severity of the disease on the same fruits, by using the scale. When the scale was not used, the coefficient of determination $\left(r^{2}\right)$ was 0.69 , the intercept (a) 1.42 and the angular coefficient $(b) 0.77$, while by using the scale these parameters were $0.84,1.59$ and 0.84 , respectively. The scale was shown to be appropriate for severity assessment of Alternaria Brown Spot on citrus fruits.

Additional keywords: Alternaria alternata, pathometry, disease evaluation

A Mancha Marrom de Alternaria (MMA) é causada por Alternaria alternata. A doença provoca desfolha, seca de ramos e queda de frutos em tangerinas e seus híbridos (1). Os sintomas em frutos são lesões deprimidas, com o centro marrom-escuro e circundadas por halo alaranjado. O centro das lesões pode, dependendo da variedade e espécie, tornar-se corticoso e saliente, formando pústulas, que se destacam com facilidade (1).

O objetivo do trabalho foi elaborar e validar uma escala diagramática para a avaliação da Mancha Marrom de Alternaria em frutos cítricos.Na confecção da escala, 200 frutos de tangor 'Murcott' e 200 de tangerina 'Ponkan', com sintomas característicos de MMA foram fotografados visando determinar a forma, a distribuição e a freqüência das lesões, assim como quantificar a área lesionada dos frutos. Os níveis mínimo e máximo da escala foram determinados pelos níveis reais observados no campo. A quantificação da severidade desses frutos foi feita no programa Quant versão 1.0.1 (2). A validação da escala diagramática foi feita por quatro avaliadores. Os avaliadores estimaram a severidade da doença de 79 fotos de frutos com sintomas, inicialmente, sem o auxílio da escala e, posteriormente, com o uso da mesma. A análise dos dados foi realizada por regressões lineares entre a severidade real e a severidade estimada pelos avaliadores. A acurácia das estimativas de cada avaliador foi determinada pelo teste $t$ ao intercepto $(a)$ e ao coeficiente angular $(b)$. A precisão das estimativas foi avaliada pelo coeficiente de determinação $\left(r^{2}\right)$ e pela variação dos erros absolutos. A reprodutibilidade das avaliações foi verificada por regressão linear, considerando as estimativas do melhor avaliador e as estimativas dos demais avaliadores.

A escala diagramática foi representada por dois tipos de lesões: 


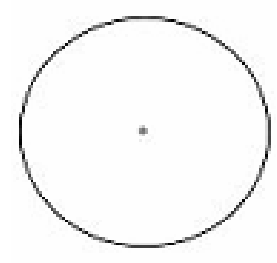

$0,1 \%$

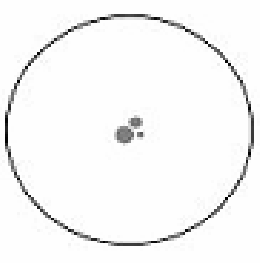

$1 \%$

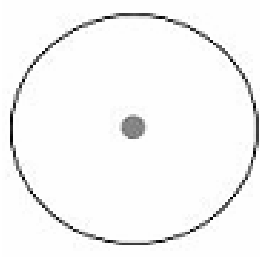

$1 \%$

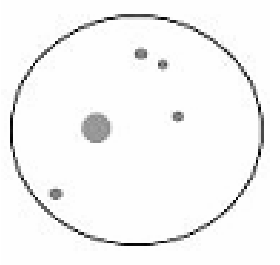

$2,5 \%$

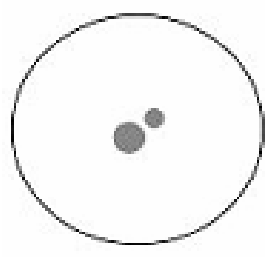

$2,5 \%$

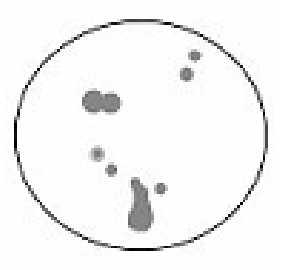

$5 \%$

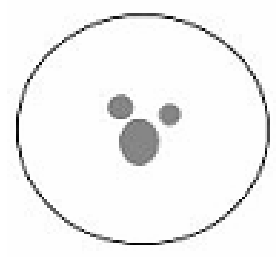

$5 \%$

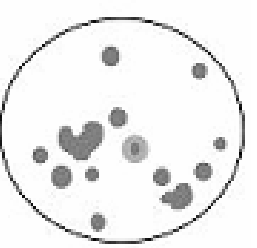

$11 \%$

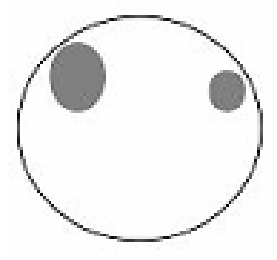

$10 \%$

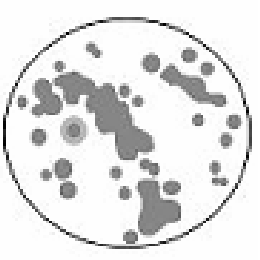

$25 \%$

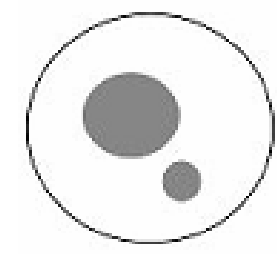

$17 \%$

Figura 1. Escala diagramática para avaliação da Mancha Marrom de Alternaria dos citros (Alternaria alternata) em frutos de tangor 'Murcott', com seis níveis de severidade da doença para pequenas lesões circulares ou áreas irregulares (linha superior) e cinco níveis para lesões grandes e circulares (linha inferior).

Tabela 1. Intercepto $(a)$, coeficiente angular $(b)$ e coeficiente de determinação $\left(r^{2}\right)$ das regressões lineares entre severidades real e estimada por cada avaliador separadamente e por todos juntos sem e com o uso da escala diagramática.

\begin{tabular}{|c|c|c|c|c|c|c|}
\hline \multirow{3}{*}{ Avaliador } & \multicolumn{3}{|c|}{ Sem escala } & \multicolumn{2}{|c|}{ Com escala } & \multirow{3}{*}{$\mathbf{r}^{2}$} \\
\hline & \multicolumn{2}{|c|}{ Parâmetros } & \multirow{2}{*}{$\mathbf{r}^{2}$} & \multicolumn{2}{|c|}{ Parâmetros } & \\
\hline & $\mathrm{a}$ & $\mathrm{b}$ & & $\mathrm{a}$ & $\mathrm{b}$ & \\
\hline A & 0,47 & 0,99 & 0,82 & 0,58 & 1,00 & 0,86 \\
\hline B & $1,55^{*}$ & $0,61 *$ & 0,85 & $2,56 *$ & $0,80 *$ & 0,82 \\
\hline $\mathrm{C}$ & $0,74 *$ & $0,63 *$ & 0,78 & $1,56^{*}$ & $0,84 *$ & 0,86 \\
\hline $\mathrm{D}$ & $2,81 *$ & $0,85^{*}$ & 0,74 & $1,67 *$ & $0,90 *$ & 0,82 \\
\hline Todos & 1,42 & $\mathbf{0 , 7 7}$ & 0,69 & 1,59 & 0,84 & 0,84 \\
\hline
\end{tabular}

*indica valores de $a$ significativamente diferentes de zero e de $b$ significativamente diferentes de 1,0 a $5 \%$ de probabilidade pelo teste $t$.

pequenas circulares ou irregulares, com 6 níveis de severidade $(0,1$; 1,$0 ; 2,5 ; 5,0 ; 11,0$ e $25,0 \%$ ) e grandes e circulares com 5 níveis de severidade $(1,0 ; 2,5 ; 5,0 ; 10,0$ e 17,0\%) (Figura 1$)$.

$\mathrm{Na}$ primeira etapa da validação, os avaliadores apresentaram precisão, com $r^{2}$ variando de 0,74 a 0,85 . O coeficiente angular $(b)$ da reta de regressão entre severidade real e severidade estimada foi igual a $1,0(p<0,05)$ em apenas um caso. Os outros três avaliadores subestimaram a doença. $O$ coeficiente angular da reta de regressão de todas as avaliações realizadas sem a escala foi de 0,23 . Com o uso da escala, o coeficiente de determinação foi de 0,16 (Tabela 1 ). Todos os avaliadores ficaram mais acurados, com o uso da escala, diminuindo a tendência de subestimar a severidade da doença.

O valor do intercepto obtido pela regressão conjunta dos dados de todos os avaliadores sem a utilização da escala diagramática foi 1,42, enquanto que com o uso da escala foi 1,59 (Tabela 1). Três avaliadores melhoraram a precisão na estimativa da severidade da doença com o auxílio da escala diagramática, haja vista que maiores valores do $r^{2}$ foram obtidos com o uso da escala (Tabela 1). Estimativas da severidade efetuadas sem o auxílio da escala diagramática, quando os dados de todos os avaliadores foram agrupados explicaram $69 \%$ da variação na mensuração da doença, enquanto que com o uso da escala a variação foi de $84 \%$ (Tabela 1 ).

Weber \& Jorg (3) relatam que o $r^{2}$ para equações de regressão que relacionam estimativas visuais com níveis reais variaram 70 a $95 \%$ entre avaliadores. Os $r^{2}$ das regressões lineares estimadas entre a severidade do melhor avaliador e a dos demais avaliadores foram satisfatórios $\left(r^{2}>0,8\right)$. Em relação aos valores de $b$ e $a$ ocorreu resultado semelhante com a utilização da escala diagramática.

A escala diagramática desenvolvida neste trabalho é recomendada como uma ferramenta de auxílio na estimativa da severidade da Mancha Marrom de Alternaria em frutos de tangerinas e seus híbridos.

\section{AGRADECIMENTOS}

A Fundação de Amparo à pesquisa do Estado de São Paulo pela colaboração na realização deste trabalho (proc. n. 03/06171-0).

\section{REFERÊNCIAS BIBLIOGRÁFICAS}

1. Feichtenberger, E. Manejo integrado das principais doenças fúngicas dos citros no Brasil. Fitopatologia Brasileira, Brasília, v.28, supl., p.231, 2003.

2. Vale, F. X. R.; Fernandes Filho, E. I.; Liberato, J. R. Quantificação de doenças. Quant v.1.0.1, Universidade Federal de Viçosa, Viçosa, 2001.

3. Weber, G.E.; Jorg, E. Errors in disease assessment a survey. Phytopathology, St. Paul, v.81, n.10, p.1238, 1991. 